\title{
Los discursos científicos en disputa al interior del campo jurídico*
}

\section{The scientific discourses discussed inside the legal field}

\author{
Manuela Graciela González** \\ Marina Laura Lanfranco Vázquez ${ }^{\star \star \star}$
}

\section{Resumen:}

Dentro de nuestro proyecto, que transita el campo jurídico, ${ }^{1}$ Ixs investigadorxs nos encontramos con la necesidad de escribir en revistas jurídicas para cumplir una de las etapas de este proceso. Desde allí, nos interrogamos acerca de si escribimos por necesidad, por obligación o en busca de legitimar nuestros hallazgos. En ese derrotero, ¿cómo se compatibilizan nuestros deseos?

Al introducirnos en estas interrogantes, surgen preguntas más específicas sobre nuestro hacer cotidiano: ¿qué rol cumplen las revistas científicas jurídicas en la construcción, difusión y retroalimentación del conocimiento jurídico?, ¿escribimos de una forma estandarizada, normada, preestablecida y desde la práctica aprehendida de "saber escribir" y "saber dónde enviar" nuestros aportes?

\footnotetext{
* Artículo de investigación Número Especial de Aniversario. Recibido el 17 de abril de 2020 y aceptado para su publicación el 16 deoctubre de 2020.

** Docente en la Facultad de Ciencias Jurídicas y Sociales de la Universidad Nacional de La Plata, Argentina. / manuelagracielagonzalez@gmail.com / https://orcid.org/0000-0002-7015-481X

*** Docente en la Facultad de Ciencias Jurídicas y Sociales de la Universidad Nacional de La Plata, Argentina. I mllanfranco@yahoo.com.ar / https://orcid.org/0000-0002-1684-0333
}

1 "En el campo jurídico se desarrolla una lucha por el monopolio del derecho a decir derecho, es decir, por establecer cuál es la buena distribución (nomos) o el buen orden. Lucha en la que se enfrentan agentes investidos de una competencia inseparablemente social y técnica, consistente en lo esencial en la capacidad socialmente reconocida de interpretar (de manera más o menos libre o autorizada) un cuerpo de textos que consagran la visión legítima, recta, del mundo social”. Bourdieu, Pierre y Teubner, Gunther, La Fuerza del derecho. Bogotá, Siglo del Hombre Editores/Facultad de Derecho de la Universidad de Los Andes/Ediciones Uniandes, 2000. p. 160. 
Por otra parte, nos interesa recuperar del imaginario académico qué grado de veracidad tiene la siguiente afirmación: la legitimación recibida a través de la publicación nos garantizará que algunxs decisorxs jurídicxs y políticxs tendrán acceso a este material para fundamentar sus trabajos, sentencias y proyectos legislativos. Además, ¿qué literatura eligen Ixs juecxs para fundamentar sus sentencias?

Partiendo de estas interrogantes y de otras que irán surgiendo de nuestra línea argumentativa fortalecida por el material bibliográfico que utilizaremos, pretendemos reflexionar críticamente desde nuestra doble función de integrantes de una revista y de personas que debemos/queremos escribir en revistas científicas tratando de hacerlo desde una distancia óptima que nos permita capturar lo novedoso de este trabajo para fortalecer nuestro campo.

PALABRAS CLAVE: campo jurídico - revistas científicas - investigadorxs conocimiento jurídico

\section{Abstract:}

For our project, within the legal field, we, the researchers need to write in law journals in order to fulfil one of the stages of this process. Therefore, we ask ourselves if writing is a need, an obligation or what allows us to legitimate our findings. Consequently, how can we achieve our goals?

Once we consider this subject, other more specific questions about our daily job emerge: What is the role of scientific and law journals in the construction, diffusion and feedback of legal knowledge? And, Do we write in a standardized, normalized and pre-established way and from the learnt practice of "writing well", or "sending our work to the best magazine"?

Additionally, we are interested in recovering the academic imaginary. How true is the following affirmation: the legitimation received through publishing will guarantee that some lawyers and politicians will have access to this material in 
order to justify their work, judgements and legislative projects. And, which literature do judges choose?

Starting with these questions and other questions which will emerge following our argumentative supported by the bibliography we will use, we aim to reflect critically from our double position of members of a magazine and people who must and want to write in scientific magazines and trying to do so from a proper distance which allows us to capture what is novel in this work in order to strengthen our field.

Key words: legal field - scientific journals - researchers - legal knowledge

\section{De interrogante en interrogante}

Este trabajo surge en respuesta a una convocatoria que realizara la Revista IUS, la cual nos resultó una interesante provocación para reflexionar críticamente sobre qué hacemos y cómo lo hacemos, y poner en debate esas reflexiones. Con la finalidad de ampliar nuestra visión del tema, decidimos consultar a las personas que trabajan en nuestra revista y ampliarla incluyendo a otras personas que lo hacen en otras revistas jurídicas de la misma facultad.

Esta decisión —creemos — nos ayudó a realizar una más amplia reflexión crítica sobre nuestro trabajo. Para ello, diseñamos un breve cuestionario, el cual fue enviado a investigadorxs ${ }^{2}$ que se encuentran relacionadxs con el mundo de las revistas jurídicas, en distintos roles, directorxs, integrantes de comités editoriales, editorxs de sección, entre otros roles en la Facultad de Ciencias

\footnotetext{
2 "Siendo el lenguaje un modo de representar(nos) el mundo y dado que el idioma castellano nombra con el género masculino a la pluralidad, dicha generalización puede constituir un modo de ocultar las tensiones y violencias inherentes al patriarcado como estructura de dominación y organización de las identidades en géneros dicotómicos. Entendiendo que en el lenguaje se cristalizan las desigualdades, las relaciones de poder y jerarquía, elegimos escribir con X, imprimiendo el carácter inconcluso del lenguaje y planteando una incógnita intentando avanzar en un modo de comunicación que no discrimine. Sin perjuicio de ello, se ha priorizado la utilización de universales que no signifiquen un menoscabo de colectivos invisibilizados históricamente por el Derecho, teniendo en consideración el uso del sistema Braille". Información contenida en la primera página del Programa de Estudio y de Examen del Espacio Curricular de Sociología Jurídica - Cátedra II, según el Plan de Estudios VI (Res 336/13), en cumplimiento con la resolución del Honorable Consejo Directivo 82/15. Recuperado de: http://campus.jursoc.unlp.edu.ar/course/view.php?id=595
} 
Jurídicas y Sociales de la Universidad Nacional de La Plata (FCJyS-UnLP), en Argentina. $^{3}$

Debido al tamaño de la muestra, a las respuestas recibidas les otorgamos un número y, en algún caso, utilizamos sus frases, sin revelar la identidad de las personas que las contestaron. Estos insumos nos fueron útiles para ampliar nuestra mirada y describir en este ensayo ${ }^{4}$ algunas interrogantes que nos permitan refrendar o contrarrestar argumentaciones. ${ }^{5}$ En nuestro doble (o triple) rol de integrantes de una revista jurídica, investigadoras y, en este momento, autoras del presente ensayo, nos incluimos dentro de la muestra.

El proceso de construcción del conocimiento implica una bidireccionalidad, es decir, supone la interacción permanente entre las categorías de Ixs investigadorxs y las categorías de Ixs actorxs, adoptando un carácter reflexivo a lo largo de todas las etapas de la investigación; "a través de este incesante ida y vuelta, el investigador puede acceder a nuevos significados, a nuevas relaciones contextuales $\mathrm{y}$, por lo tanto, a nuevas interpretaciones". ${ }^{6}$ De este modo, la construcción de conocimiento entre el sujeto cognoscente y el sujeto conocido ${ }^{7}$ es cooperativa y flexible, y permite producir "nuevos conceptos y conexiones explicativas sobre la base de los presupuestos iniciales, ahora reformulados y enriquecidos por categorías de los actores y sus usos contextualizados en la vida social".8

\footnotetext{
${ }^{3}$ El cuestionario fue enviado de forma virtual y se han recibido las respuestas de la misma forma, teniendo en consideración el contexto de público conocimiento de crisis sanitaria y de afección global que nos encontramos transitando en el presente. La República Argentina ha dispuesto el aislamiento social preventivo y obligatorio para disminuir el contagio de Covid-19 desde el día viernes 20 de marzo de 2020, de acuerdo con lo establecido por el Decreto 297/2020. Recuperado de: https://www.boletinoficial.gob.ar/detalleAviso/primera/227042/20200320.

${ }^{4}$ Un ensayo científico es un texto escrito en prosa, relativamente breve y dedicado a un tema en particular relacionado con las ciencias. En él se expresa un punto de vista personal sobre el tema abordado, basado en una información recogida y presentada de manera objetiva. Lxs autorxs desarrollan las ideas de forma organizada y utilizando un lenguaje técnico.

${ }^{5}$ Los cuestionarios diseñados para la elaboración del presente y contestados por las personas consultadas (datos primarios) se encuentran disponibles en caso de ser requeridos.

${ }^{6}$ Guber, Rosana, El salvaje metropolitano. Reconstrucción del conocimiento social en el trabajo de campo, Buenos Aires, Paidós, 2005, p 81.
}

7 Vasilachis de Gialdino, Irene, "La investigación cualitativa", Estrategias de investigación cualitativa, Barcelona, Gedisa, 2006.

${ }^{8}$ Guber, Rosana, op. cit, p. 80. 
El cuestionario fue enviado inicialmente a 22 personas, de lo cual se obtuvieron las respuestas de 15 de ellas, 10 mujeres y 5 varones. Algunas forman parte de la Revista Derecho y Ciencias Sociales ${ }^{9}$ y desempeñan diferentes roles, otras están a cargo de la dirección o son integrantes del comité editorial, y el resto son editorxs con menor autonomía de decisión con respecto a la política de la revista. El rol de editorxs de sección se nutre de investigadorxs en formación, becarixs del sistema científico universitario en Argentina.

Ninguna de las personas que trabaja en la revista recibe retribución por su tarea, y la unidad académica de la cual depende tampoco brinda subsidios para sostenerla ni muestra especial interés por su difusión. La universidad sí ha llevado adelante una política activa de brindar formación y elementos técnicos para el crecimiento de las revistas de este tipo.

Esta composición nos muestra que quienes integran la revista conocen y valoran la investigación jurídica y aceptan las reglas que ella establece, destacando que para promocionar es necesaria la publicación en revistas de esta calidad.

Al analizar las respuestas decidimos diferenciarlas realizando un corte relacionado con las etapas de formación científica en que se encuentran las personas que respondieron el cuestionario (investigadorxs formadxs, en formación y becarixs). Esto - estimamos- nos permitió tener una visión más precisa del campo de análisis.

En la primera pregunta del cuestionario nos interrogamos si cuando escribimos sobre nuestros avances en investigación y enviamos nuestros trabajos a una revista jurídica prestigiosa lo hacemos por necesidad, por obligación o para legitimar nuestros hallazgos. De las 15 respuestas recibidas, la mayoría ha acordado que lo hacen por necesidad y para legitimar hallazgos en investigación.

Al relacionar las respuestas con la etapa de formación en la investigación antes referida, observamos que en la segunda etapa (investigadorxs en formación) se incluyen más frecuentemente discursos relacionados con la discusión de hallazgos con otrxs profesionales que estén trabajando en líneas similares. La

\footnotetext{
${ }^{9}$ La Revista Derecho y Ciencias Sociales, del Instituto de Cultura Jurídica y de la Maestría en Sociología Jurídica, de la UNLP, de publicación semestral on line, se encuentra disponible en: https://revistas.unlp.edu.ar/dcs/index
} 
franja de investigadorxs formadxs refiere con mayor frecuencia a mostrar resultados, compartir hallazgos, pesquisas logradas.

Solo tres respuestas han indicado un motivo distinto a los preestablecidos en el cuestionario. Una de ellas se concatena con la publicación por obligación, por tornarse un requisito esencial para acreditar la producción científica frente a los órganos de financiación de la ciencia, destacando la importancia de comunicar avances o resultados de investigación. ${ }^{10}$

Otra de las personas consultadas ha indicado la necesidad de poner en la agenda de este tipo de revistas los cambios paradigmáticos que se están verificando donde se incluya la perspectiva de género. ¿Podría pensarse así a la revista como un vehículo para discutir "imaginarios androcéntricos"? ${ }^{11}$ Esta última respuesta nos genera nuevas interrogantes: ¿podríamos pensar a la revista jurídica como un espacio o campo de disputa paradigmática?

¿Las revistas científicas deben/deberían ser receptoras de artículos científicos que discutan desde diferentes paradigmas o deberían tener una ideología explícita? En algunas de las fundamentaciones a las respuestas dadas se han incluido dimensiones como evaluación, reconocimiento y transferencia. Respecto de la evaluación, se podría subdividir en dos aspectos: por un lado, el sistema científico requiere este tipo de publicaciones para la acreditación de la producción individual; y por otro, la garantía de imparcialidad que otorga una evaluación absolutamente exógena bajo doble nudo ciego.

Respecto a la trasferencia, se ha destacado la importancia de formar parte de las discusiones de determinada problemática específica, de ser leído por pares y por lectorxs en general.

Tabla 1. Necesidad, obligación, legitimación de hallazgos

\begin{tabular}{|l|l|l|l|l|l|l|l|l|l|l|l|l|l|l|l|}
\hline & 1 & 2 & 3 & 4 & 5 & 6 & 7 & 8 & 9 & 10 & 11 & 12 & 13 & 14 & 15 \\
\hline
\end{tabular}

10 De la comunicación personal a partir del cuestionario contestado en la entrevista número 8.

${ }^{11}$ De la comunicación personal a partir del cuestionario contestado en la entrevista número 10. 


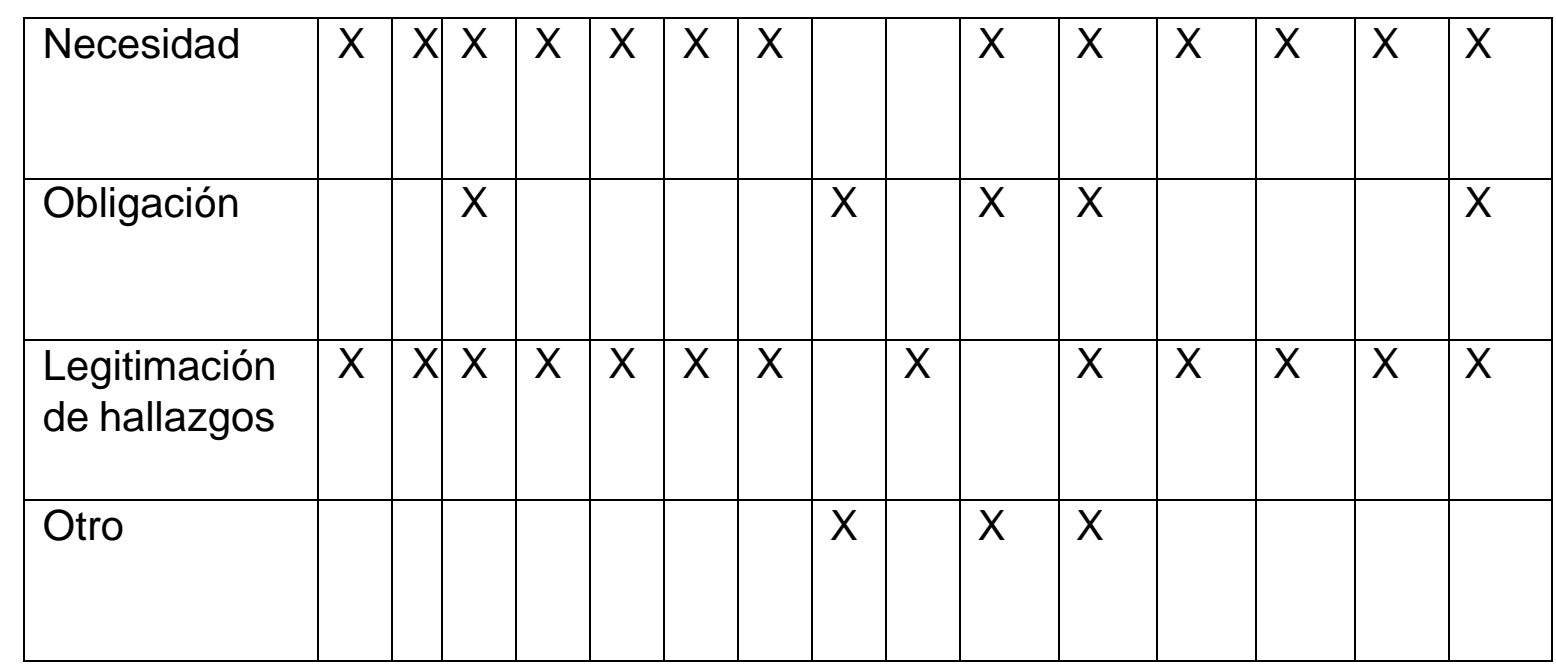

Fuente: Elaboración propia con base en los cuestionarios diseñados para esta publicación.

En la segunda cuestión nos preguntábamos sobre el rol que cumplen las revistas científicas jurídicas. Ha predominado la difusión o divulgación de la producción de conocimiento científico, pero han aparecido otros aspectos destacables en las respuestas brindadas: legitimación, puesta en debate de un área específica del conocimiento, actualización de perspectivas, estándares o problemáticas jurídicas que también se canalizan o se posicionan a través de las revistas.

Un renglón aparte merece la referencia respecto de la evaluación o el sentido de "control en la forma de hacer ciencia", ${ }^{12}$ lo cual se valida de manera externa y, por tanto, otorga legitimidad. Pero frente a la misma pregunta, se abre un opuesto respecto a ese "control", que puede dar cuenta de un conocimiento de elite y, por tal razón, endogámico. ${ }^{13}$

Tabla 2. Rol de las revistas científicas.

\begin{tabular}{|l|l|l|l|l|l|l|l|l|l|l|l|l|l|l|l|}
\hline & 1 & 2 & 3 & 4 & 5 & 6 & 7 & 8 & 9 & 10 & 11 & 12 & 13 & 14 & 15 \\
\hline $\begin{array}{l}\text { Divulgación c / } \\
\text { difusión }\end{array}$ & $\mathrm{X}$ & $\mathrm{X}$ & $\mathrm{X}$ & $\mathrm{X}$ & & & & $\mathrm{X}$ & $\mathrm{X}$ & & & & $\mathrm{X}$ & $\mathrm{X}$ & $\mathrm{X}$ \\
\end{tabular}

${ }^{12}$ Comunicación personal a partir del cuestionario contestado en la entrevista número 1.

${ }^{13}$ Comunicación personal a partir del cuestionario contestado en la entrevista número 10. 


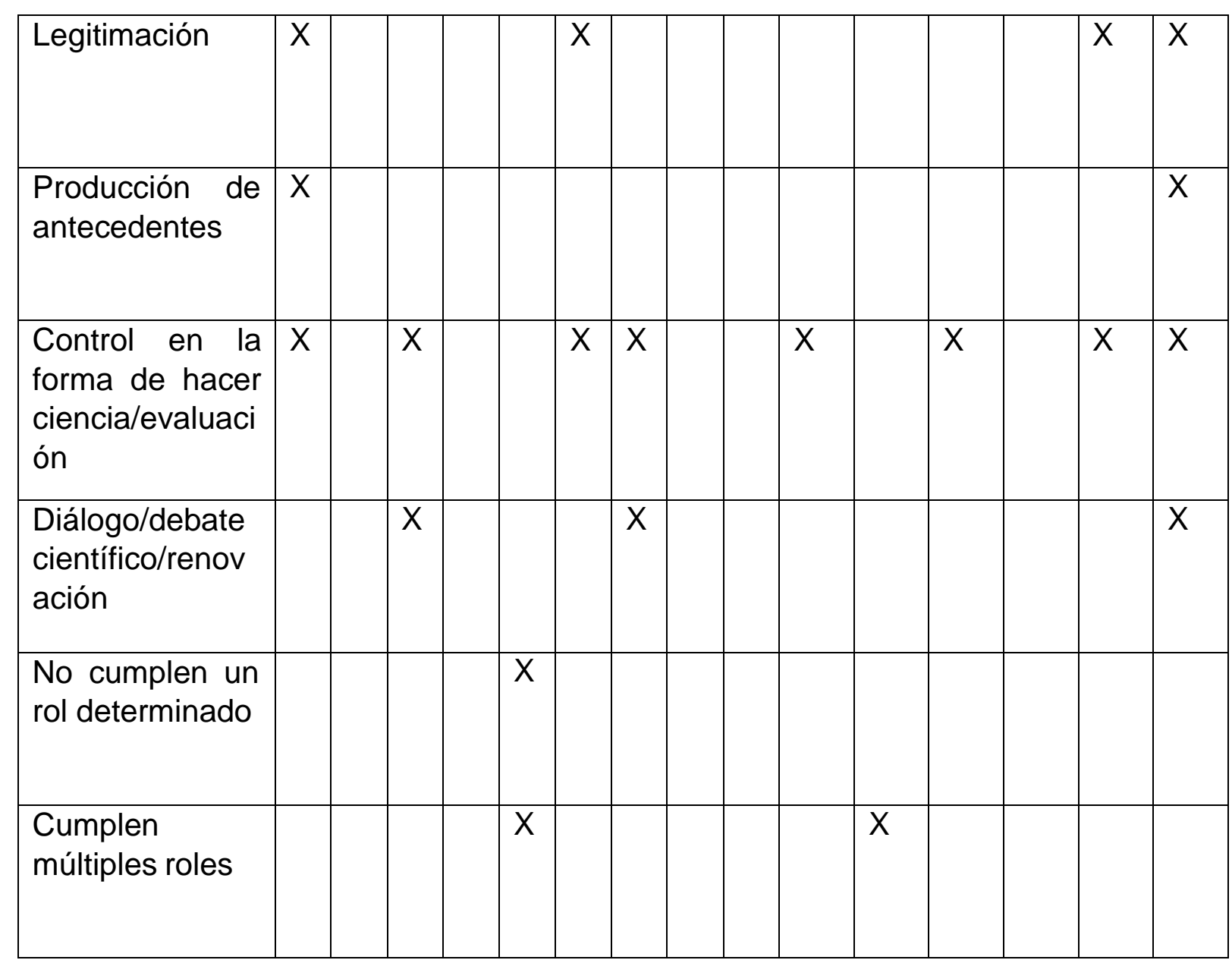

Fuente: Elaboración propia con base en los cuestionarios diseñados para esta publicación.

La tercera de las cuestiones versaba sobre el entrenamiento que obtiene la persona al enviar un artículo a una revista relacionado con las prácticas de saber escribir y saber dónde enviar.

Tabla 3. Saber escribir y saber dónde enviar.

\begin{tabular}{|l|l|l|l|l|l|l|l|l|l|l|l|l|l|l|l|}
\hline & 1 & 2 & 3 & 4 & 5 & 6 & 7 & 8 & 9 & 10 & 11 & 12 & 13 & 14 & 15 \\
\hline Saber escribir & $\mathrm{X}$ & $\mathrm{X}$ & $\mathrm{X}$ & $\mathrm{X}$ & & $\mathrm{X}$ & $\mathrm{X}$ & $\mathrm{X}$ & $\mathrm{X}$ & & $\mathrm{X}$ & $\mathrm{X}$ & $\mathrm{X}$ & $\mathrm{X}$ & $\mathrm{X}$ \\
& & & & & & & & & & & & & & & \\
\hline
\end{tabular}




\begin{tabular}{|l|l|l|l|l|l|l|l|l|l|l|l|l|l|l|l|}
\hline $\begin{array}{l}\text { Saber dónde } \\
\text { enviar }\end{array}$ & $\mathrm{X}$ & $\mathrm{X}$ & $\mathrm{X}$ & $\mathrm{X}$ & $\mathrm{X}$ & $\mathrm{X}$ & $\mathrm{X}$ & $\mathrm{X}$ & $\mathrm{X}$ & & & $\mathrm{X}$ & $\mathrm{X}$ & $\mathrm{X}$ & $\mathrm{X}$ \\
\hline No contesta & & & & & & & & & & $\mathrm{X}$ & & & & & \\
\end{tabular}

Fuente: Elaboración propia con base en los cuestionarios diseñados para esta publicación.

Saber dónde enviar permite identificar algunos obstáculos, por ejemplo, conocer si el proceso de evaluación de la revista es demorado. Esto suele ser un inconveniente para compatibilizar con los tiempos para acreditar la producción científica ante las agencias que financian las investigaciones (individuales o grupales). Las particularidades formales de cada revista a veces tornan engorroso el proceso de envío. ${ }^{14}$

Saber dónde enviar nos permite, a su vez, identificar algunos beneficios, tales como mejorar la calidad de la producción científica, tanto por la misma práctica de la escritura como por las devoluciones de las evaluaciones realizadas. Se constituye en un interesante proceso cuando, una vez publicado, adquiere mayor difusión y, al mejorar la calidad, es apreciado por la comunidad científica. También permite conocer las distintas revistas, sus tradiciones, sus universos y sus índices de impacto, parámetros que dan posibilidad de decidir dónde enviar nuestros trabajos.

Aparece aquí una diferencia marcada entre las personas consultadas, sean investigadorxs formadxs o en formación. En el primer caso, además de la calidad de la revista, a veces se prefieren las tradiciones, y en el segundo está más clara la indexación como un parámetro definitorio del saber enviar, para que redunde positivamente en los antecedentes.

Una de las respuestas dadas se refirió a la inconveniencia de utilizar artículos científicos para argumentar posiciones de las partes en los pleitos o fundar las sentencias, ya que considera que este tipo de escritos es de otro orden. El artículo científico se refiere a un recorte de la realidad con pautas metodológicas

${ }^{14}$ Comunicación personal a partir del cuestionario contestado en la entrevista número 2. 
muy exactas, y en la praxis la argumentación jurídica tiende a "convencer" o buscar apoyatura a la versión de una de las partes en cuestión. ${ }^{15}$

La última pregunta de nuestro cuestionario se refería a la problemática de la difusión de la ciencia jurídica y a qué literatura considera que eligen Ixs juecxs para fundamentar sus sentencias. Según las respuestas dadas, se utilizan muy frecuentemente revistas jurídicas que no son científicas, denominadas por algunas de las personas consultadas como tradicionales y que se distribuyen y se comercializan teniendo como destinatarixs lectorxs de la profesión liberal o la administración de justicia; sentencias previas (propias o de pares), legislación, recopilaciones de jurisprudencia y legislación, doctrina de autores del campo de la dogmática jurídica. Se menciona, a su vez, el formato libro; con menor incidencia, revistas especializadas.

La necesidad de definir conceptualmente la ciencia jurídica para luego problematizar sobre ella ha sido expresada por una de las personas consultadas como crítica a la formulación de la pregunta realizada en el cuestionario. ${ }^{16}$ Aquí reaparece otra interrogante: ¿es más prestigioso escribir en revistas científicas en ciencias sociales que en revistas científicas jurídicas?

¿Se asocian las revistas jurídicas con producciones que analizan normas, doctrina y jurisprudencia sin el uso de la rigurosidad del método científico?

Por otro lado, se destacó que la literatura jurídica científica se encuentra en creciente difusión e ingresa la variable "ideológica" de Ixs actores jurídicos en esa selección. ${ }^{17}$

Una de las respuestas se introdujo en otro orden de ideas, no se refiere al formato de publicaciones a las que apelan Ixs juezxs, sino a la utilización frecuente, en el campo de investigación específico, de la persona consultada de "discursos psi". ${ }^{18}$

\footnotetext{
${ }^{15}$ Comunicación personal a partir del cuestionario contestado en la entrevista número 6.

${ }^{16}$ Comunicación personal con base en el cuestionario contestado en la entrevista número 5.

17 Idem.

${ }^{18}$ Comunicación personal a partir del cuestionario contestado en la entrevista número 7.
} 
También se han diferenciado el fuero y las instancias de la administración de justicia, los volúmenes de trabajo y "quienes" escriben efectivamente las sentencias, entrando en otro nivel de complejidad en este análisis.

\section{Hacia nuevos interrogantes}

Este ensayo nos ha permitido dialogar con nuestros pares y ampliar la mirada hacia nuevas dimensiones que alimentan la necesidad de ahondar la reflexión crítica sobre lo que hacemos, que posiblemente fructificará en nuevos trabajos de mayor profundidad.

La ciencia jurídica como tal, con sus bordes imprecisos, nos interroga sobre la necesidad o no de delimitar los bordes, ya que se encuentra permeada por problemáticas jurídicas, sociales y culturales que la atraviesan y la cuestionan. Por ello, nos desafía a realizar un trabajo mancomunado desde la interdisciplina, en diálogo con otras ciencias sociales ${ }^{19} \mathrm{y}$ desde la interseccionalidad ${ }^{20}$ como perspectiva epistemológica.

Es decir, se podría pensar desde la interdisciplina como aporte para proponer soluciones al modelo autorreferencial del derecho y una manera de sobrepasar el enfoque puramente normativo (normológico o dogmático), integrando otras dimensiones que permitan entender los fenómenos sociales, analizar y aplicar las normas en relación con la realidad social, pero nada dicen acerca de la adopción de la perspectiva de género, ${ }^{21}$ que se encuentra ausente, en gran medida, de los discursos jurídicos.

No obstante los avances hacia estas direcciones, resulta importante mencionar, a su vez, que estas perspectivas y búsquedas se encuentran ausentes, también y sobre todo, en la formación de grado de quienes luego se desempeñarán como

\footnotetext{
${ }^{19}$ Galletti, Hilda Gabriela, "Interdisciplina, género y derecho”, en González, Manuela G.; Miranda, Marisa y Zaikoski Biscay, Daniela, Género y derecho, La Pampa, EdUNLPam, 2019, pp. 83-97; Pezzeta, Silvina, "¿Qué investigar sobre la enseñanza del derecho en las facultades de abogacía? El techo de cristal en la investigación sociojurídica", Academia. Revista sobre Enseñanza del Derecho (Argentina), núm. 18, año 9, 2011, pp. 59-75.

20 Viveros Vigoya, Mara, "La interseccionalidad: una aproximación situada a la dominación", Debate Feminista (Colombia), vol. 52, octubre de 2016, pp.1-17.
}

${ }^{21}$ Galleti, Hilda Gabriela, op. cit. 
actorxs clave en la praxis jurídica, tanto en la administración de justicia y en la profesión libre como en la academia (docencia e investigación) y en distintos espacios de la gestión pública y privada, reproduciendo un discurso jurídico hegemónico con una gran impronta dogmática. ${ }^{22}$

Por ello, desde la interseccionalidad se ofrecen otras comprensiones de las problemáticas sociales que ponen en discusión el discurso jurídico hegemónico y que suman elementos de integración y amplitud comprensiva a los debates hacia el interior del campo jurídico. ${ }^{23}$ En tal sentido, las revistas jurídicas deberían ser receptoras de artículos científicos que discutan desde distintos paradigmas.

Los criterios de evaluación deberían ser compartidos por las revistas científicas jurídicas. Hay diferencia entre la escritura en ciencias sociales y la escritura en ciencias jurídicas. Al no tener la investigación jurídica una tradición histórica extensa la hace menos legitimada al interior del campo jurídico. Quizá alguno de los aportes específicos del campo y que pueden brindar las revistas de ciencia jurídica sea publicar resultados de investigaciones empíricas interdisciplinarias y que esos hallazgos puedan ser legitimados por Ixs actores judiciales. ${ }^{24}$

Existen dimensiones de trasferencia del conocimiento entre pares científicos o transferencia a estudiantes de grado, posgrado y a funcionarixs o abogadxs litigantes.

¿Lxs investigadorxs vemos a Ixs decisorxs judiciales y abogadxs con dificultades para comprender la complejidad de la construcción del conocimiento científico en el campo, marcando una tensión entre ciencia y ciencias jurídicas? Esto nos conmina a pensar si quienes investigamos pertenecemos a un campo diferente en el sentido de Bourdieu, y si ese campo presenta un mayor prestigio - que, de alguna manera, si es citado, pierde parte de su situación de privilegio- 0

${ }^{22}$ González, Manuela G., "El género como recurso de la enseñanza legal", en González, Manuela G.; Miranda, Marisa y Zaikoski Biscay, Daniela, Género y derecho, La Pampa, EdUNLPam, 2019, pp. 271-291; González, Manuela G. y Cardinaux, Nancy (comp.), Los actores y las prácticas. Enseñar y aprender derecho en la UNLP, La Plata, Edulp, 2010.

${ }^{23}$ La Barbera, María Caterina, "Interseccionalidad", Eunomía. Revista en Cultura de la Legalidad (España), núm. 12, abril a septiembre. DOI: https://doi.org/10.20318/eunomia.2017.3651; Viveros Vigoya, Mara, op. cit.

${ }^{24}$ Pezzeta, Silvina, op. cit. 
podemos sostener que los datos sobre la realidad que el sistema científico en este campo pueda brindar pueden ser valorados como elementos que mejoren las argumentaciones de Ixs decisorxs jurídicxs.

¿El campo de recontextualización del conocimiento, en términos de Bernstein, podría pensarse hacia dentro de la administración de justicia ${ }^{25}$ En tal sentido, ¿de qué forma sería posible esta transferencia o recontextualización?

Esta tensión presente entre el campo científico y el político en textos weberianos podría ser trasladada del científico al decisor jurídico que en realidad explicita poco su concepción política pero, como lo muestra la lectura académica con rigurosidad científica, deja sus marcas en la sentencia judicial.

Con la obligatoriedad de la Ley Micaela ${ }^{26}$ para todas las personas que trabajan en relación con el Estado en Argentina, resulta interesante mencionar que solo una de las respuestas alude a incorporar como obligatoria la transversalidad de la perspectiva de género tanto en la producción como en la difusión. ¿Esto obligaría a modificar qué se investiga, cómo y para qué?

Debemos aclarar, sobre este último punto, que ninguna pregunta estaba direccionada hacia el género; no obstante, al menos cuatro cuestionarios fueron respondidos utilizando lenguaje inclusivo.

¿Es importante para las revistas jurídicas la divulgación hacia el interior del campo judicial y a quienes trabajan en el ejercicio de la profesión jurídica o estas publicaciones buscan principalmente ser legitimadas como producciones del campo de la investigación científica?

Lxs investigadorxs que saben dónde enviar sus artículos tiene una herramienta que marca diferencia, ¿esa información la comparten intergeneracionalmente? Nos preguntamos también si forma parte de un perfil individualista y competitivo que se fomenta por parte de las agencias de investigación que acreditan la

\footnotetext{
25 Idem, citando a Bernstein, p. 62.

${ }^{26}$ La denominada Ley Micaela establece que "la capacitación obligatoria en la temática de género y violencia contra las mujeres para todas las personas que se desempeñen en la función pública en todos los niveles y jerarquías en los tres poderes Ejecutivo, Legislativo y Judicial" (art. 1 de la Ley Nacional sobre la Capacitación Obligatoria en Género, para todas las personas que integran los tres poderes del Estado, bajo el № 27.499 del año 2018). Esto se dio como resultado de la movilización social producida tras el femicidio de la adolescente Micaela García, acontecido en 2017.
} 
producción científica personal para ingresar o permanecer en términos de accesibilidad en esos saberes expertos de saber escribir y saber enviar. ¿Cómo se democratizan esos saberes?

Se plantea la necesidad de pensar en términos de democratización del conocimiento y de socialización de los detalles de cómo hacerlo efectivo intergeneracionalmente entre investigadorxs formados, en formación y becarixs que puedan trabajar integradamente, de manera horizontalizada y colaborativa. De alguna forma estamos pensando en términos de transversalizar el conocimiento.

Paulo Freire nos interpela sobre "¿cómo construir la pedagogía de la movilidad?", ${ }^{27}$ lo cual nos sugiere pensar en términos de movilidad hacia el interior de la ciencia jurídica, en sus posibilidades de movimiento transversal donde aparezcan estas interrelaciones discursivas antes expresadas en las encuestas y que hemos recorrido a través de la bibliografía analizada.

¿Las revistas científicas jurídicas pueden proponerse como campo de democratización de esos saberes? ¿Podría pensarse como interrogantes posibles y en términos epistemológicos sobre nuestro hacer investigativo que al publicar en una revista jurídica científica se ponga en común, discusión o debate un nuevo conocimiento más equitativo, con perspectiva de género e igualitario? O, en términos de Santos, ${ }^{28}$ ¿es posible pensar que a través de las publicaciones en las revista jurídicas científicas se abone a un discurso emancipatorio del derecho?

Estas ideas van fructificando en relación con los discursos jurídicos que se encuentran en disputa hacia el interior del campo jurídico, con el fin de abrir puertas hacia nuevas posibilidades y comprensiones.

\section{Referencias bibliográficas}

${ }^{27}$ Freire, Paulo, El grito manso, Buenos Aires, Siglo XXI, 2003, p. 52.

${ }^{28}$ Santos, Boaventura de Souza, Sociología jurídica crítica. Para un nuevo sentido común en el derecho, Madrid, Trotta/lsla, 2009. 
Bourdieu, Pierre y Teubner, Gunther, La fuerza del derecho, Bogotá, Siglo del Hombre Editores/Facultad de Derecho de la Universidad de Los Andes/Ediciones Uniandes, 2000.

Freire Paulo, El grito manso, Buenos Aires, Siglo XXI, 2003.

Galletti, Hilda Gabriela, "Interdisciplina, género y derecho", en González, Manuela G.; Miranda, Marisa y Zaikoski Biscay, Daniela, Género y derecho, La Pampa, EdUNLPam, 2019.

González, Manuela G., "El género como recurso de la enseñanza legal", en González, Manuela G.; Miranda, Marisa y Zaikoski Biscay, Daniela, Género y derecho, La Pampa, EdUNLPam, 2019.

González, Manuela G. y Cardinaux, Nancy (comp.), Los actores y las prácticas. Enseñar y aprender Derecho en la UNLP, La Plata, Edulp, 2010.

Guber, Rosana, El salvaje metropolitano. Reconstrucción del conocimiento social en el trabajo de campo, Buenos Aires, Paidós, 2005.

La Barbera, María Caterina, "Interseccionalidad", Eunomía. Revista en Cultura de la Legalidad (España), núm. 12, abril a septiembre. DOI: https://doi.org/10.20318/eunomia.2017.3651

Pezzeta, Silvina, “¿Qué investigar sobre la enseñanza del derecho en las facultades de abogacía? El techo de cristal en la investigación sociojurídica", Academia. Revista sobre Enseñanza del Derecho (Argentina), núm. 18, año 9, pp. 59-75.

Santos, Boaventura de Souza, Sociología jurídica crítica. Para un nuevo sentido común en el derecho, Madrid, Trotta/lsla, 2009.

Vasilachis de Gialdino, Irene, "La investigación cualitativa", Estrategias de investigación cualitativa, Barcelona, Gedisa, 2006. 
Viveros Vigoya, Mara, "La interseccionalidad: una aproximación situada a la dominación", Debate Feminista (Colombia) vol. 52, octubre de 2016, pp. 1-17.

\section{Norma legal}

Ley Micaela sobre la Capacitación Obligatoria en Género para todas la personas que integran los tres poderes del Estado № 27.499 del año 2018, Publicada en el Boletín Nacional con fecha 10 de enero de 2019. Disponible en http://servicios.infoleg.gob.ar/infoleglnternet/anexos/315000319999/318666/norma.htm

Decreto $297 / 2020$ sobre el Aislamiento Social Preventivo y Obligatorio publicado en el Boletín Oficial el día 20 de marzo de 2020. Disponible en https://www.boletinoficial.gob.ar/detalleAviso/primera/227042/20200320. 